Relations industrielles

Industrial Relations

\title{
1978 : perspectives économiques et gestion sociale de \\ l'entreprise, Paris, Institut de Gestion sociale, 1978, 234 pp.
}

\section{Dimitri Weiss}

Volume 33, numéro 2, 1978

URI : https://id.erudit.org/iderudit/028874ar

DOI : https://doi.org/10.7202/028874ar

Aller au sommaire du numéro

Éditeur(s)

Département des relations industrielles de l'Université Laval

ISSN

0034-379X (imprimé)

1703-8138 (numérique)

Découvrir la revue

Citer ce compte rendu

Weiss, D. (1978). Compte rendu de [1978 : perspectives économiques et gestion sociale de l'entreprise, Paris, Institut de Gestion sociale, 1978, 234 pp.] Relations industrielles / Industrial Relations, 33(2), 366-367.

https://doi.org/10.7202/028874ar

Tous droits réservés (C) Département des relations industrielles de l'Université Laval, 1978
Ce document est protégé par la loi sur le droit d'auteur. L'utilisation des services d'Érudit (y compris la reproduction) est assujettie à sa politique d'utilisation que vous pouvez consulter en ligne.

https://apropos.erudit.org/fr/usagers/politique-dutilisation/ 
Dans le chapitre sur «les parties en présence», il est d'abord question de quelques notions élémentaires de droit syndical, puis de la protection de la liberté syndicale et, enfin, des techniques et mécanismes concernant l'accréditation. Dans le chapitre consacré à «la négociation collective des conditions de travail », sont successivement présentées les coordonnées de la négociation, de l'arbitrage des différends, de l'exercice des moyens de pression économique, de la théorie de la convention collective et de la pratique de l'arbitrage des griefs. Une mise en contexte historique préside généralement aux explications des règles juridiques. Des observations qui vont audelà de la donnée juridique accompagnent le plus souvent ces explications. L'approche suivie par l'auteur permet de mieux situer les quelques 115 modifications apportées au Code du travail, fournit une base de discussion utile pour approfondir davantage les nouvelles règles et leur incidence sur l'ensemble du régime et, finalement, permet de constater que les «plaideurs» et les «académiciens» auront suffisamment de matières pour s'en donner à cœur joie.

Les Rapports collectifs du travail au Québec (1978), est en définitive et avant tout destiné à permettre à l'étudiant de prendre connaissance des règles de droit applicables. Par ailleurs cet ouvrage facilitera, chez les initiés, la discussion sur les changements majeurs apportés par la Loi 45. Dans l'un et l'autre cas les solutions avancées resteront des hypothèses de travail qui devront subir la «sanction de la pratique quotidienne». À ce sujet, il convient de noter une remarque fondamentale du professeur Morin: «Il est vraiment trop tôt pour élaborer une appréciation générale de l'ensemble des amendements du 22 décembre 1977. On doit d'abord laisser lel temps aux parties de les appliquer. Les lois du travail n'ont de valeur que par leurs effets réels ou virtuels. Avant la mise en application concrète, il ne s'agit que de formules, avenues ou approches plus ou moins intéressantes. On demeure encore, et malgré l'acte formel du législateur, en pleine théorie».

Université Laval
1978: perspectives économiques et gestion sociale de l'entreprise, Paris, Institut de Gestion sociale, 1978, 234 pp.

Voici que paraissent sous ce titre les Actes du premier Symposium de prospective sociale appliquée à l'entreprise, qui, organisé en septembre 1977 par l'Institut de Gestion sociale, avait réuni à Paris, dans l'enceinte du Palais de l'U.N.E..S.C.O., plus de 200 dirigeants d'entreprises et responsables de personnel.

Une première partie des interventions ont été réunies sous le titre "Certitudes et incertitudes du dirigeant» et rassemblent:

- le signataire de ces lignes, avec un exposé inaugural consacré à une "Analyse prospective des relations sociales dans l'entreprise », portant particulièrement sur le présent et l'avenir des comités d'entreprise ;

- Michel Collas, directeur général de Creusot-Loire, "Un chef d'entreprise face aux incertitudes économiques et aux objectifs sociaux»: les motivations du chef d'entreprise, pour son action sociale (l'idéalisme, la défense de la société libérale, l'efficacité de l'entreprise), action sociale et participation, moyens de la politique idoine, problèmes soulevés par la crise économique actuelle ;

- Michel Crozier, directeur du Centre de Sociologie des Organisations, «Tendances et comportements des hommes dans les organisations » : trop grande importance donnée aux motivations des individus et à une expression beaucoup trop rapide de ces motivations à travers des sondages; la situation réelle dans la majorité de la population reflète une tendance minoritaire de désaffection pour le travail ;

Une deuxième partie porte sur «Les dirigeants et la gestion sociale de leur entreprise». Y figurent:

- Philippe Dechartre et Jean-Paul Bachy: «L'influence du politique», point de vue du Rassemblement pour la République (gaullistes) et, respectivement, du Parti socialiste;

- Table ronde sur des «Expériences de gestion sociale» dans les entreprises, avec la participation de cadres dirigeants;

- Michel Logan, directeur général de l'association pour l'emploi des cadres, in- 
génieurs et techniciens, «La préparation de l'encadrement et la décentralisation des responsabilités sociales»: l'exercice de l'autorité et son style, l'effet d'entraînement par l'exemple du chef d'entreprise ;

La troisième partie était intitulée «Face à la conjoncture économique et sociale» et réunissait :

- Thierry de Montbrial, «L'analyse théorique et les évolutions possibles»: le professeur de l'École Polytechnique et chef du Centre d'analyse et de prévision du ministère des Affaires étrangères, y a fait une analyse des origines du désordre économique mondial et a dressé les conditions du redressement économique au niveau international: la réalisation d'une certaine stabilité monétaire, la théorie des locomotives (États-Unis, Allemagne fédérale, Japon), l'ordre commercial, la politique de l'énergie et de bonnes politiques nationales;

- Jean-Joseph Régent, président de la Chambre de commerce et d'industrie de Nantes, qui parla des "Solidarités et contraintes au niveau régional»

- Jean Boissonnat, rédacteur en chef de la revue L'Expansion, "Les comportements des chefs d'entreprise» à la suite d'une crise dont nous ne sommes pas prêts de sortir;

- François Bloch-Lainé qui en tira la conclusion «Face à la conjoncture économique et sociale»: progresser socialement ne veut pas dire seulement améliorer la condition propre des travailleurs, mais encore et surtout, perfectionner la marche des organisations productives à toutes fins; car c'est en cela que les actions économiques et sociales sont liées, tant au plan «macro» que « micro».

La quatrième partie, intitulée "Quelles réponses pour l'entreprise?», mit face à face :

- Xavier Mallet, président-directeur général des Pompes Guinard, sur "L'évolution du rôle et de la légitimité des chefs d'entreprise et des responsables des ressources humaines", qui préconisa, notamment, les solutions à nos maux mises en œuvre dans l'entreprise qu'il dirige: petites unités à l'échelle humaine, si possible à la campagne; les réunions du personnel, qui puisse s'exprimer sur ses propres pro- blèmes; des équipes opérationnelles, constituées ad hoc pour la solution des diverses questions soulevées dans la vie de l'entreprise; les équipes autonomes;

- Pierre Cabanes, directeur des relations du travail au ministère du Travail, «Quelles réponses pour l'entreprise aux nouvelles obligations légales, notamment du bilan social?»: évocation des textes législatifs récents en matière d'accidents du travail, de retraite, de durée du travail, de repos compensateur, d'amélioration des conditions de travail, de bilan social;

- Yvon Chotard, vice-président du Conseil national du patronat français, «L'innovation sociale est-elle possible dans la conjoncture économique des prochains mois?» Oui si deux conditions préalables sont remplies: l'établissement d'une politique claire de rémunérations et l'implication de l'encadrement.

L'allocution finale était prononcée par le ministre du travail, Christian Beullac, qui insista sur l'insertion des jeunes, grâce à un meilleur rapprochement de l'éducation et du travail, au combat contre les rigidités et les corporatismes, à l'amélioration des conditions de travail.

Il est difficile d'analyser et de porter un jugement global sur un ouvrage de ce genre. Les contributions, inégales, les unes plus improvisées, les autres plus élaborées, sont juxtaposées et partent dans tous les sens. Pour les participants au symposium, comme pour les lecteurs, chacun y (aura) trouve (ra) ce qu'il y apporte. Mais un panorama des idées en matière sociale et économique à la veille de 1978 en France méritait d'être fait, et, sous cet angle, l'apport de ces Actes apparaît positif et, parfois, séduisant.

Dimitri WEISS

Université de Paris I Panthéon-Sorbonne

La pratique des relations paritaires dans l'entreprise, par Étienne Ricard, Paris, Collection Centor, Entreprise moderne d'édition, 1977, 252 pp.

Cet ouvrage, écrit par un praticien, s'adresse avant tout aux praticiens dans le but de les aider dans leur travail quotidien. 